\title{
Analysis of Pore Formation and Protein Translocation Using Large Biological Nanopores
}

\author{
Hirokazu Watanabe, ${ }^{\dagger}$ Alberto Gubbiotti, ${ }^{\ddagger}$ Mauro Chinappi, ${ }^{\S}$ Natsumi Takai, $^{\dagger}$ Koji Tanaka, ${ }^{\prime \prime}$ \\ Kouhei Tsumoto," and Ryuji Kawano*,† \\ ${ }^{\dagger}$ Department of Biotechnology and Life Science, Tokyo University of Agriculture and Technology, 2-24-16 Naka-cho, Koganei, \\ Tokyo 184-8588, Japan \\ ${ }^{\ddagger}$ Department of Mechanical and Aerospace Engineering, Sapienza University of Rome, Via Eudossiana 18, Rome 00184, Italy \\ ${ }^{\S}$ Department of Industrial Engineering, University of Rome Tor Vergata, Via del Politecnico 1, Rome 00133, Italy \\ "Department of Chemistry and Biotechnology, School of Engineering, The University of Tokyo, Bunkyo-ku, Tokyo 113-8656, Japan
}

\section{Supporting Information}

ABSTRACT: This paper describes the analysis of pore formation and detection of a single protein molecule using a large nanopore among five different pore-forming proteins. We demonstrate that the identification of appropriate pores for nanopore sensing can be achieved by classifying the channel current signals and performing noise analysis. Through these analyses, we selected a perforin nanopore from the membrane attack complex/perforin superfamily and attempted to use it to detect the granzyme B protein, a serine protease. As a result, we found that granzyme B might pass through the perforin nanopore if it adopts an unfolded structure. Our proposed analytical approach should be useful for exploring several types of nanopore as large biological nanopores other than $\alpha$ hemolysin.

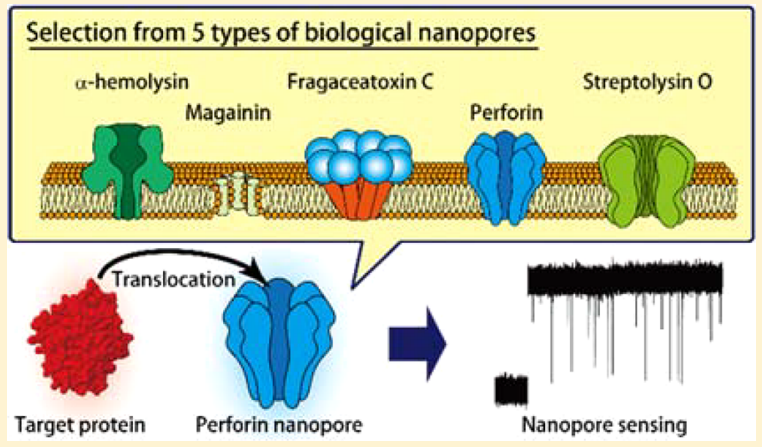

Target protein Nanopore sensing
$\mathrm{N}$ anopore sensing has emerged as a unique method for detecting single molecules. One of the most important and unique features of this method is that it involves electrical measurement at the single-molecule level of the ion currents through a nanopore; when one of the target molecules binds or passes through the pore, the current clearly decreases, which is represented as the blocking current. ${ }^{1}$ This blocking current and the duration of analyte binding or translocation reveal the chemical identity and properties at the single-molecule level. In addition, the frequency of current blocking reflects the concentration of analytes, indicating that this approach can also be used for quantification. ${ }^{2-4}$ Although this method is powerful for single-molecule detection, its selectivity essentially depends on the size of analytes and the nanopore. For example, $\alpha$-hemolysin $(\alpha \mathrm{HL})$ is generally used as a biological nanopore, with a pore of $1.4 \mathrm{~nm}$ in diameter at its narrowest point. ${ }^{5-10}$ This is highly compatible for detecting single-stranded DNA (ssDNA) because its diameter is approximately $1 \mathrm{~nm}$. As such, DNA detection is a particularly attractive field of nanopore study. ${ }^{11,12}$ On the other hand, for the detection of small molecules, a molecular binder that binds to the interior of $\alpha \mathrm{HL}$ and narrows its size has been proposed. ${ }^{1}$ Alternatively, DNA aptamer has been used as a component to bind to the target molecule. ${ }^{3}$ The specific sequence of ssDNA can recognize and bind to the target, forming a complex that is larger in size; as a result, the complex is captured into the $\alpha \mathrm{HL}$ nanopore. ${ }^{3}$
However, larger molecules, such as proteins, for which the diameter is normally $3-5 \mathrm{~nm}$, cannot be detected by an $\alpha \mathrm{HL}$ nanopore because they are too large.

To overcome this limitation, larger nanopores can be fabricated by using solid-state nanopores. The pore size of a solid-state nanopore can be controlled by the fabrication procedure, for which protein detection at the single-molecule level has also been attempted. ${ }^{2,13-17}$ With regard to biological nanopores, protein detection still remains a challenge, although several candidate nanopores other than $\alpha \mathrm{HL}$ have been reported (Table 1). At present, the major issues with biological nanopores are as follows: (1) Most biological pores are too small to detect proteins. (2) A stable lipid bilayer is required because large pores readily result in rupture of the lipid membrane. Against this background, in this study, we explored appropriate nanopores for detecting protein molecules from five pore-forming protein families, namely, the hemolysin (toxin), antimicrobial peptide (antimicrobial), actinoporin (toxin), membrane attack complex/perforin (MACPF) (immunity), and cholesterol-dependent cytolysins (toxin) families (Figure 1). To investigate the pore-forming activity of these proteins, we employed a high-throughput system that can

Received: April 27, 2017

Accepted: October 5, 2017

Published: October 5, 2017 
Table 1. Biological Nanopores That Have Been Used for Nanopore Sensing ${ }^{a}$

\begin{tabular}{|c|c|c|c|c|}
\hline Nanopore & Diameter $[\mathbf{n m}]$ & Shape & Target molecules & Ref.[PDBID] \\
\hline MspA & 1.2 & & ssDNA, dsDNA & {$[1 \mathrm{UUN}]^{20,21}$} \\
\hline a-hemolysin & 1.4 & & $\begin{array}{l}\text { Metal ion, small organics, RNA, ssDNA, Amino acid, Unfold- } \\
\text { ed protein, Polymers, Gold nanoparticle }\end{array}$ & {$[7 \mathrm{AHL}]^{5,11}$} \\
\hline Aerolysin & $1-1.7$ & & Peptide, Protein & {$[5 \mathrm{JZT}]^{22,23}$} \\
\hline Omp & $\sim 2$ & & Protein, Antibody & {$[2 \mathrm{~F} 1 \mathrm{C}]^{24,25-26}$} \\
\hline FhuA & $\sim 2.4$ & & Protein, Protein-ssDNA, polymers & {$[1 \mathrm{BY} 3]^{27,28}$} \\
\hline VDAC & $2.5-3$ & & Tubulin, Small organics, dsDNA & {$[2 \mathrm{JK} 4]^{29,30}$} \\
\hline SP1 & $\sim 3$ & & ssDNA & {$[1 \mathrm{TR} 0]^{31,32}$} \\
\hline Phi29 & 3.6 & & ssDNA, dsDNA, Thioester antibody & {$[1 \mathrm{FOU}]^{33,34}$} \\
\hline ClyA & $3.3-4.2$ & & ssDNA, Protein & {$[2 \mathrm{WCD}]^{35,36}$} \\
\hline
\end{tabular}

${ }^{a} \alpha \mathrm{HL}$ is the most common biological nanopore used for nanopore sensing. The diameter of these nanopores is $<5 \mathrm{~nm} .{ }^{19}$ All protein structures are from Protein Data Bank (PDB).

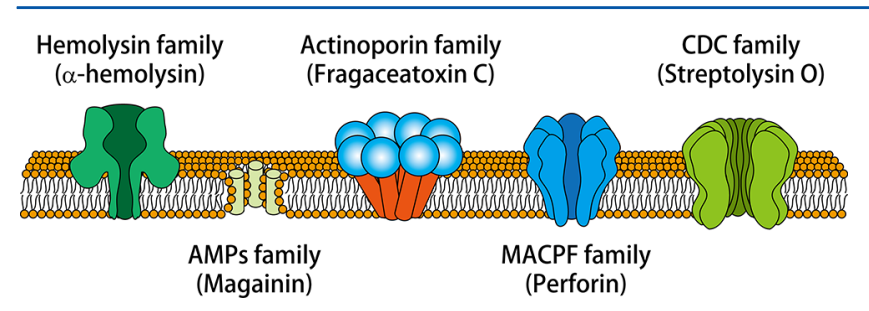

Figure 1. Representation of five types of nanopore reconstituted in a lipid bilayer. We selected five nanopores from different pore-forming protein families and evaluated each pore's properties.

prepare stable and reproducible lipid bilayers using a droplet contact method (Figure 2). ${ }^{18}$ Through our investigation, we revealed the stability of the open-pore state and the pore size of five nanopores, from which we propose a method of estimating two different pore-forming behaviors by analyzing the noise of the open-channel current. Finally, using a perforin (PFN) nanopore from the MACPF family, we analyzed the translocation of a single protein, granzyme B (GZB), passing through it.

\section{EXPERIMENTAL SECTION}

Reagents and Chemicals. The reagents used in this study were as follows: 1,2-diphytanoyl-sn-glycero-3-phosphocholine (DPhPC; Avanti Polar Lipids, AL, USA); 1,2-dioleoyl-snglycero-3-phosphocholine (DOPC; Avanti Polar Lipids); 1,2dioleoyl-sn-glycero-3-phosphoethanolamine (DOPE; Avanti Polar Lipids); 1,2-dioleoyl-sn-glycero-3-phospho-(1'-rac-glycerol) (DOPG; Avanti Polar Lipids); sphingomyelin (SM; brain, porcine, Avanti Polar Lipids); cholesterol (Avanti Polar Lipids); 


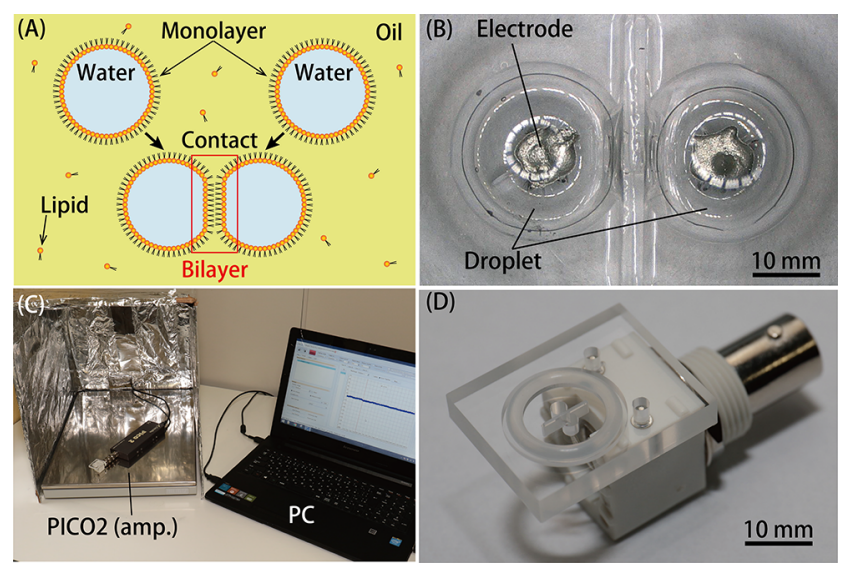

Figure 2. (A) Lipid bilayer prepared using the droplet contact method. (B) A photograph of two chambers for formation of a lipid bilayer (top view). (C) A photograph of the setup of a device connected with a laptop PC. (D) A photograph of the device for measuring channel currents.

n-decane (Wako Pure Chemical Industries, Ltd., Osaka, Japan); 3-morpholinopropane-1-sulfonic acid (MOPS; Nacalai Tesque, Kyoto, Japan); $\mathrm{KCl}$ (Nacalai Tesque); $\mathrm{CaCl}_{2}$ (Nacalai Tesque); dithiothreitol (DTT; Wako Pure Chemical Industries); $\alpha$ hemolysin ( $\alpha \mathrm{HL}$; Sigma-Aldrich); magainin 1 (Mag; LKT Laboratories Inc., MN, USA); perforin (PFN; Kamiya Biomedical Company, WA, USA); streptolysin O (SLO; Bio Academia, Osaka, Japan); granzyme B (GZB; R\&D System Inc., MN, USA). FraC was obtained from the Tsumoto laboratory (University of Tokyo). Buffered electrolyte solutions were prepared from ultrapure water, which was obtained from a Milli-Q system (Millipore, Billerica, MA, USA).

Lipid Bilayer Preparation and Reconstitution of PoreForming Protein. Lipid bilayers were prepared by the "droplet contact method" ${ }^{37}$ using a device fabricated with microfabrication technology. In this method, two lipid monolayers contact and form a bilayer as shown in Figure 2A. First, $2.3 \mu \mathrm{L}$ of lipid/oil solution was dropped into each chamber. Next, $4.7 \mu \mathrm{L}$ of the buffer solution was dropped into both chambers. Then, two lipid monolayers contacted and formed a bilayer. Pore-forming proteins were added to only one side of the chambers and spontaneously reconstituted in lipid bilayer. The detailed procedures of this system have been previously reported. ${ }^{37}$

We applied various buffers and lipid compositions when performing the measurements on each nanopore. $\alpha \mathrm{HL}$ was dissolved at $0.33 \mathrm{nM}$ in buffer solution including $1 \mathrm{M} \mathrm{KCl}$ and $10 \mathrm{mM}$ MOPS. Mag was dissolved at $10 \mu \mathrm{M}$ in buffer solution including $200 \mathrm{mM} \mathrm{KCl}$ and $10 \mathrm{mM}$ MOPS. FraC was dissolved at $10 \mathrm{nM}$ in buffer solution including $1 \mathrm{M} \mathrm{KCl}$ and $10 \mathrm{mM}$ MOPS. PFN was dissolved in buffer solution including $140 \mathrm{mM}$ $\mathrm{KCl}, 0.1 \mathrm{mM} \mathrm{CaCl}_{2}$, and $10 \mathrm{mM}$ MOPS. SLO was dissolved at $16 \mathrm{nM}$ in buffer solution including $140 \mathrm{mM} \mathrm{KCl}, 10 \mathrm{mM}$ DTT, and $10 \mathrm{mM}$ MOPS. For all measurements, the buffer solution was $\mathrm{pH}$ 7.0. The lipid composition for each measurement is shown below. $\alpha \mathrm{HL}$ and PFN were reconstituted in DPhPC membrane. Mag was reconstituted in DOPG/DOPE $=1: 3$ (mol/mol) membrane. FraC was reconstituted in DPhPC/SM $=5: 1(\mathrm{~mol} / \mathrm{mol})$ membrane. SLO was reconstituted in $\mathrm{DPhPC} /$ cholesterol $=1: 1(\mathrm{~mol} / \mathrm{mol})$ membrane. Within a few minutes of adding the buffer solution, two lipid monolayers connected and formed a lipid bilayer, and pore-forming proteins formed nanopores by reconstitution. When the lipid bilayer ruptured during this process, we reformed it by tracing with a hydrophobic stick (typically using pipet chips) at the interface of the drops. Through this process, no influence of contamination at the droplet fuse was observed in this study. ${ }^{38}$

Channel Current Measurements and Data Analysis. Channel currents were monitored using a PICO patch clamp amplifier (Tecella, Foothill Ranch, CA, USA) connected to an $\mathrm{Ag} / \mathrm{AgCl}$ electrode in each chamber. These electrodes were in each droplet when we added the solution into the chambers. Nanopores formed in the bilayers allowed ions to pass through them under the voltage gradient, so we obtained signals of the channel current. These signals were detected using a $4-\mathrm{kHz}$ low-pass filter at a sampling frequency of $20 \mathrm{kHz}$. Analysis of channel signals was performed using pCLAMP ver. 10.7 (Molecular Devices, CA, USA).

Signal Classification. We classified the current signals into three types by reference to the shape of the time-current traces. First, when step-like signals were observed, in which the current sharply increased and then plateaued, we counted one step of the current increase as one signal. Second, we regarded a current increase with continuous fluctuation as multilevel signals and counted the period from the start to the finish of the current increase as one signal. The end of the current increase involved a return to $0 \mathrm{pA}$ or an increase larger than the measurement limit. Third, when spike signals occurred that involved short increases in current that then momentarily returned to $0 \mathrm{pA}$, we counted a series of such events at a similar amplitude as one signal. ${ }^{39}$

Calculation of Pore Diameter and the Number of Monomers. To estimate the pore size and monomer assembly state of each nanopore, we analyzed the current conductance of the step-like signals. The Hille equation was used in the pore diameter estimation. ${ }^{40}$

$$
R=\left(l+\frac{\pi r}{2}\right) \frac{\rho}{\pi r^{2}}
$$

Here, $r$ is the pore radius, $\rho$ is the resistivity of the buffered solution, $l$ is the length of the pore $(20 \mathrm{~nm}$ for PFN from the pore model $^{41}$ and $7 \mathrm{~nm}$ from the thickness of the lipid bilayer for other pores), and $R$ is the resistivity of the pore. $R$ is calculated as $V / I$, where $I$ is the current through the pore and $V$ is the applied voltage between two chambers $(100 \mathrm{mV})$. We calculated the pore diameter of FraC using the equation for a cone-like nanopore. ${ }^{42}$

Using the pore diameter and monomer size from the Protein Data Bank (PDB), the number of assembling monomers was mathematically calculated as follows:

$$
d=a\left(\frac{1}{\sin (\pi / n)}-1\right)
$$

where $d$ is the pore diameter, $a$ is the width of a peptide monomer from the PDB, and $n$ is the number of assembling monomers. The arc model is presented in the Supporting Information.

Granzyme B Detection and Analysis. To achieve the detection of proteins using the PFN nanopore, we added granzyme $\mathrm{B}(\sim 5 \mathrm{~nm}, \mathrm{GZB})$ at a concentration of $1 \mu \mathrm{M}$ to the cis side. (In this experiment, the cis side was where the potential of $+100 \mathrm{mV}$ was applied and the trans side was grounded.) GZB is a serine protease that acts in the human immune system by inducing the apoptosis of target cells. 


\section{RESULTS AND DISCUSSION}

Classification of Current Signals and Estimation of Pore Diameter. Five different pore-forming proteins could be reconstituted in a planar lipid bilayer with the individual reconstituted conditions (see Experimental Section), and the channel currents were observed. Whereas a step-like signal is imperative for nanopore measurement, other shapes of current signals appeared in all nanopore measurements. Therefore, we classified these signals based on the previously proposed classification ${ }^{43}$ as shown in Figure 3A. Three different current

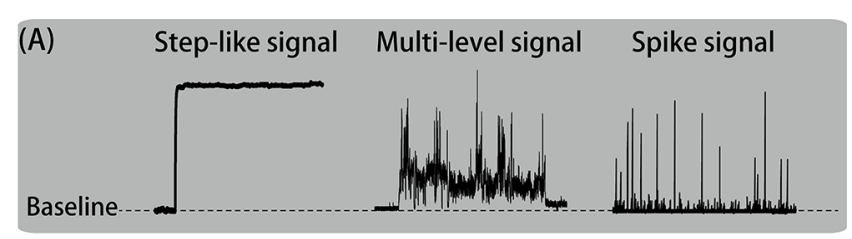

(B)
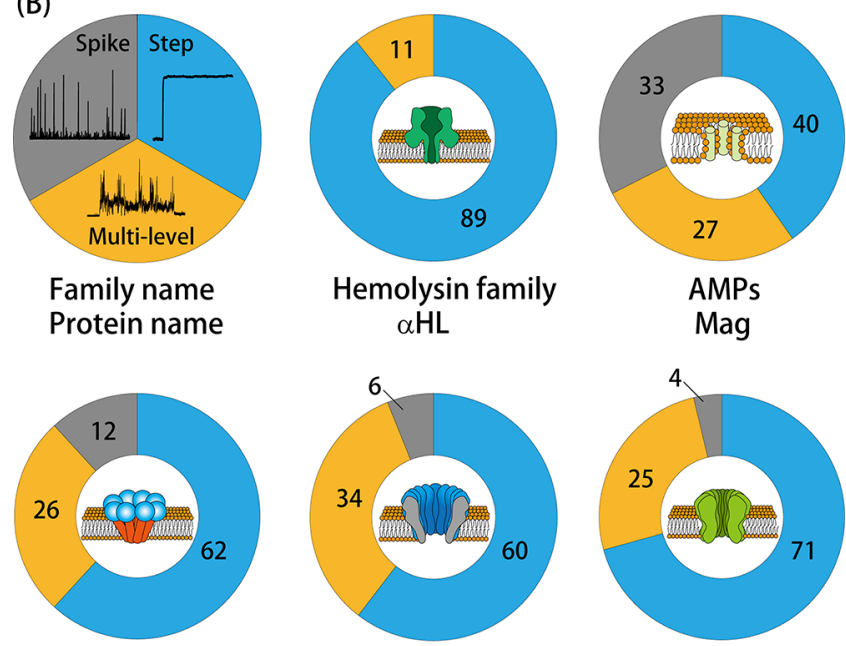

Actinoporin FraC
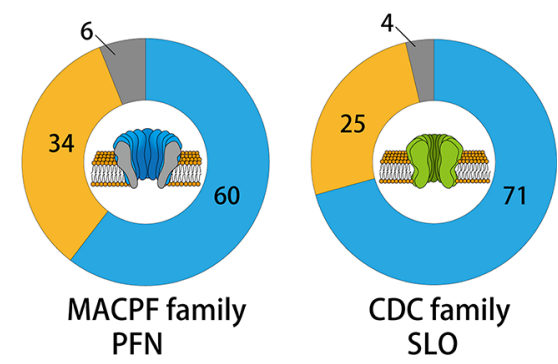

Figure 3. (A) Schematic of three typical types of signals. (B) Signal classification of five types of nanopore. The numbers in the circular graphs indicate the percentages of each signal among all signals $(n=$ 77-215). Nanopores that show a high proportion of step-like signal are suitable for nanopore sensing.

signals, namely, step-like, multilevel, and spike, were classified from four different protein measurements except that of $\alpha \mathrm{HL}$ because $\alpha \mathrm{HL}$ showed only two types of signals (Figure S1). Studies involving measurement of the current of synthetic channels or antimicrobial peptides previously reported that this variance of the current signals reflects the variance of the mechanism by which defects in the membrane arise: ${ }^{39}$ (1) The step-like signal reflects robust and stable pore formation, resulting in a constant current level. This mechanism is also known as a "barrel-stave model" in the field of antimicrobial peptides (AMPs). ${ }^{44}$ (2) The multilevel signal reflects unstable current fluctuation after pore opening, indicating that the shape and size of pores could vary after pore formation. This model can be applied to random pore defects or the "toroidal model" of AMPs, which form pores via the assembly of monomers and lipids. ${ }^{44}$ (3) The spike signal implies the short open-closed transition of a pore caused by membrane penetration with monomers or the "carpet model" of AMPs. ${ }^{44}$ For successful nanopore sensing, a step-like signal should dominate. The ratio of the appearance of each signal type is shown in Figure 3B.
The proportion of the step-like signal in $\alpha \mathrm{HL}$ was $89 \%$, which was the highest value among them; this is the key reason for the domination of $\alpha \mathrm{HL}$ in the nanopore measurements. The order of the other proportions was $\mathrm{SLO}>\mathrm{FraC}>\mathrm{PFN}$, which were all $>60 \%$. In contrast, the proportion for Mag was $40 \%$; this low step-like proportion indicated that Mag is not suitable for use in nanopore sensing. The high proportions for $\alpha \mathrm{HL}$, SLO, FraC, and PFN reflect that they have large outer membrane domains, which stabilize the assembling state with monomers and result in stable pores. ${ }^{42}$ On the other hand, Mag is a relatively small molecule and only has a transmembrane region without the outer membrane domains. Consequently, the monomer of Mag is more likely to form pores with other monomers and lipids. ${ }^{45}$ This caused the proportion of step-like signals for Mag to be the lowest. In addition, the spike signal for Mag was relatively large, suggesting that this small peptide can penetrate through the lipid membrane due to its low molecular weight and fewer hydrophilic domains. From these results, we concluded that SLO, FraC, and PFN can be applied to nanopore sensing.

The pore diameter was calculated using the conductance of the step-like signals and a theoretical model, as listed in Table 2. In this estimation, the Hille model ${ }^{40}$ was used for the

Table 2. Properties of the Reconstituted Nanopores ${ }^{a}$

\begin{tabular}{|c|c|c|c|c|c|}
\hline name & family & $\begin{array}{c}\text { pore } \\
\text { diameter }^{b} \\
{[\mathrm{~nm}]}\end{array}$ & $\begin{array}{l}\text { pore range } \\
{[\mathrm{nm}]}\end{array}$ & $\begin{array}{c}\text { stoichiometry } \\
{[\mathrm{mer}]}\end{array}$ & $\begin{array}{c}\text { ratio of } \\
\text { step } \\
\text { signal } \\
{[\%]}\end{array}$ \\
\hline$\alpha \mathrm{HL}$ & hemolysin & 1.4 & 1.4 & 7 & 89 \\
\hline Mag & AMPs & 1.1 & $0.5-3$ & $4-10$ & 40 \\
\hline FraC & actinoporin & $1.7,2.6$ & $1-4$ & $6-15$ & 62 \\
\hline $\mathrm{PFN}_{\mathrm{L}}$ & MACPF & 3.2 & $1.4-18$ & $4-21$ & 60 \\
\hline SLO & $\mathrm{CDC}$ & 1.6, 6.2, & $1-104$ & $3-116$ & 71 \\
\hline
\end{tabular}

${ }^{a}$ The pore diameter is calculated from pore conductance. Pore range indicates the minimum and maximum values of pore diameter. Stoichiometry is calculated from pore diameter. ${ }^{b}$ The most probable value of pore diameter.

estimation of SLO, PFN, and Mag because the pore shapes of SLO, PFN, and Mag were assumed to be cylindrical. Since $\mathrm{FraC}$ was assumed to feature a cone-shaped pore considering its crystal structure, Hille's equation for an asymmetrical model was used in this case. ${ }^{46}$ Additionally, the numbers of assembling monomers were also mathematically estimated using the pore diameter and width of monomers from the crystal structures. ${ }^{39}$ The histograms of the conductance and stoichiometry for each of the protein nanopores are shown in Figure S2. PFN and Mag showed a single peak, whereas SLO and FraC presented several peaks (note that a detailed discussion of the conductance of PFN is presented in the next section). This implies that there are several stable states of pore formation in SLO and FraC. As for the results of SLO, it was previously reported that the pore diameter ranges from 25 to $35 \mathrm{~nm}$ with 36- to 125 -mer of assembling monomers. ${ }^{47}$ Although these values for the SLO pore were consistent with our conductance results, small pores, of $<20 \mathrm{~nm}$, could be observed in our measurements. This small conductance implies that the monomer of SLO may participate in slight pore expansion in the process of pore assembly, and finally, it becomes a mature pore, which means a complete ringstructured pore. From the results of $\mathrm{FraC}$, it is already known from the crystal structure that the pore diameter (for the small side) is $1.6 \mathrm{~nm}$ with an 8 -mer form, ${ }^{42}$ and these values were 
compatible with the most probable peaks of the histograms (Table 2 and Figure S2).

Summarizing these results, SLO and PFN were shown to be appropriate for protein detection in terms of pore size. This is reasonable because these transmembrane proteins function in cell lysis or protein transportation. In addition, pores that are formed in these cases are relatively robust and stable, and the PFN pore has a current rectification ranging from -200 to $+200 \mathrm{mV}$, suggesting the asymmetric geometry of the pore structure (Figure S3). Considering that the diameter of the most water-soluble proteins is $3-5 \mathrm{~nm}$, we concluded that PFN would be the most appropriate nanopore for single-protein detection.

Current Noise Analysis of Channel Recording for Characterizing the Pore-Forming Process. In the current measurements of PFN, two types of step-like signal were observed: one was small $(\sim 15 \mathrm{pA})$ and showed stair-like current steps, named as $\mathrm{PFN}_{\mathrm{S}}$ (Figure 4A); the other one was large $(\sim 85 \mathrm{pA})$ and involved random steps up, named as $\mathrm{PFN}_{\mathrm{L}}$ (Figure 4B). We expected that these two distinct current steps might reflect two different pore-forming mechanisms. The small step implies that PFN monomers are added into the prospectively assembled pore one-by-one after initial pore formation, which is named the "expanding model" in this paper (Figure 4C, left). On the other hand, for the larger case, these current steps may reflect multiple pore formations of the completed pores such as $\alpha \mathrm{HL}$ pore formation, which is named the "additional model" here (Figure 4C, right). In addition, SLO also showed large or small step signals, as mentioned above. To analyze these two mechanisms, we focused on the current noise of the channel open state because the increase in the area of the interior of the pores upon their opening should differ between these mechanisms, and then, the current noise should be influenced by the area of the pore wall made of amino acid residues.

On the basis of this hypothesis, we analyzed the RMS ratios of the open channel current of PFN, SLO, and FraC and compared these with $\alpha \mathrm{HL}$ and alamethicin (Ala), as typical "additional" and "expanding" models, respectively. For $\alpha \mathrm{HL}$, it is well-known that assembled prepores bind to a lipid bilayer and form pores that are membrane-spanning. In contrast, Ala is an AMP that forms pores by assembling monomers in a lipid bilayer. The ratio of RMS can be calculated as follows:

$$
\begin{aligned}
& \mathrm{RMS}=\sqrt{\frac{\sum_{i=1}^{M}\left(x_{i}-\bar{x}\right)^{2}}{M-1}} \\
& \mathrm{RMS}_{\text {ratio }}=\frac{\mathrm{RMS}_{N}-\mathrm{RMS}_{0}}{\mathrm{RMS}_{1}-\mathrm{RMS}_{0}}
\end{aligned}
$$

where $M$ is the number of current values included in one step, $x_{i}$ is each current value included in the step, $\bar{x}$ is the mean current value of the step, $\mathrm{RMS}_{N}$ is the RMS value of the Nth step, and $\mathrm{RMS}_{0}$ is the RMS value at baseline. The relationship between the RMS noise and length of the pore circumference of $\alpha \mathrm{HL}$ and Ala is shown in Figure 4D. The slopes of $\alpha \mathrm{HL}$ and Ala were clearly different at 1.0 and 0.24 , respectively. As we expected, the slope between the $\mathrm{RMS}_{\text {ratio }}$ and length of the pore circumference is perfectly consistent with each other (Figure 4D).

Therefore, the RMS value can be used to predict whether pore formation occurs by the "additional" or the "expanding" mechanism. In the case of the "additional" model, the area of

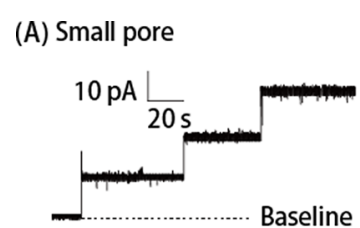

(C)

(B) Large pore
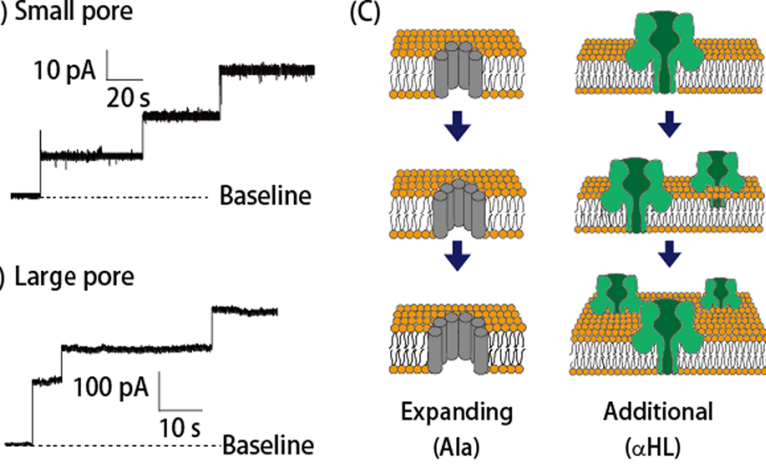

(D)
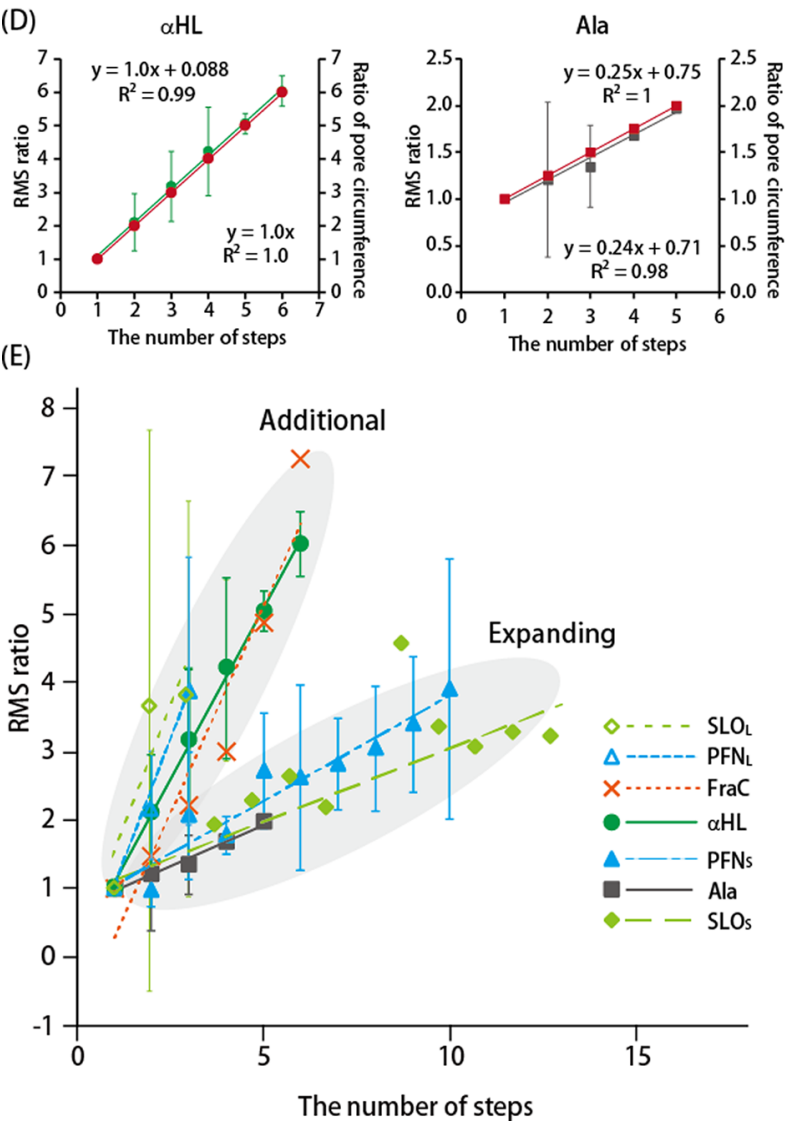

Figure 4. (A, B) Typical time-current trace of PFN small pore (A) and large pore (B) formation with $100 \mathrm{mV}$ application. (C) Two models of current increase. Ala causes a current increase by pore expansion, and $\alpha \mathrm{HL}$ causes a current increase by additional pore formation. (D) Relationship between the RMA ratio and length of pore circumference of $\alpha \mathrm{HL}$ and Ala, corresponding to additional and expanding pore formation. (E) Results of RMS analysis of nanopores. Five nanopores were divided into two models. $\mathrm{PFN}_{\mathrm{L}}, \mathrm{FraC}$, and $\alpha \mathrm{HL}$ were classified into the additional model, while $\mathrm{PFN}_{\mathrm{S}}$, Ala, and SLO were classified into the expanding model.

the pore interior will linearly increase with the reconstituted pore number. In the "expanding" model, in contrast, the area of the pore interior will increase with increasing area of the interior of the additional monomers. Figure $4 \mathrm{E}$ shows the results of the RMS value of the five different nanopores as a function of the number of current steps. The large error bars were caused by the distribution of pore size even if the same number of monomers was assembled, as observed using atomic force (AFM) or transmission electron microscopy (TEM). 
From the results of the RMS analysis, the slopes of all nanopores were close together, with values around $1.4-0.2$, as listed in Table 3. The slope of FraC, the large step of PFN

Table 3. Results of Noise Analysis and Model for Each Nanopore $^{a}$

$\begin{array}{lcc}\text { name } & \text { slope } & \text { mechanism } \\ \mathrm{SLO}_{\mathrm{L}} & 1.4 & \text { additional } \\ \mathrm{PFN}_{\mathrm{L}} & 1.4 & \text { additional } \\ \mathrm{FraC} & 1.2 & \text { additional } \\ \alpha \mathrm{HL} & 1.0 & \text { additional } \\ \mathrm{PFN}_{\mathrm{S}} & 0.31 & \text { expanding } \\ \mathrm{Ala} & 0.24 & \text { expanding } \\ \mathrm{SLOs} & 0.21 & \text { expanding }\end{array}$

${ }^{a}$ The slope of the RMS ratio differs for each nanopore. Five types of nanopores were divided into two groups depending on the slope value.

$\left(\mathrm{PFN}_{\mathrm{L}}\right)$, and SLO $\left(\mathrm{SLO}_{\mathrm{L}}\right)$ were classified into the same group as $\alpha \mathrm{HL}$ as the "additional" model. FraC forms a prepore and makes a transmembrane pore similar to $\alpha \mathrm{HL}$, as previously proposed. On the other hand, the slope of the small step of PFN $\left(\mathrm{PFN}_{\mathrm{S}}\right)$ and SLO $\left(\mathrm{SLO}_{\mathrm{S}}\right)$ corresponded to that of Ala as the "expanding" model. From these results, PFN has two poreforming mechanisms at variance with $\alpha \mathrm{HL}, \mathrm{FraC}$, and Ala. To discuss the difference between $\mathrm{PFN}_{\mathrm{L}}$ and $\mathrm{PFN}_{\mathrm{S}}$, we analyzed the duration of the pore-opening state. The median values of the duration of $\mathrm{PFN}_{\mathrm{L}}$ and $\mathrm{PFN}_{\mathrm{S}}$ were $9.7 \mathrm{~s}(n=154)$ and $15 \mathrm{~s}$ $(n=27)$, suggesting that the process of pore formation in which monomers participate was much slower than the complete pore-forming process of the "additional" model at this concentration.

Pore Formation Model and Pore Size of PFN from the Current Conductance. As mentioned above, the step currents of $\mathrm{PFN}_{\mathrm{L}}$ and $\mathrm{PFN}_{\mathrm{S}}$ were approximately $\sim 85$ and $\sim 15 \mathrm{pA}$, respectively. According to AFM or TEM observations of the PFN pore, arc-shaped incomplete pores and complete (circular) pores were observed. An AFM observation showed that the diameter of the complete pore was approximately 6.1 $\mathrm{nm}$ (Figure 5A). ${ }^{48}$ In the case of the diameter of $\mathrm{PFN}_{\mathrm{L}}$ from the current conductance, the calculated diameter using Hille's equation was approximately $3.4 \mathrm{~nm}$ (Figure $5 \mathrm{~B}$ ). This size was slightly different from the value from the AFM observation due to the cylindrical assumption of pore geometry in Hille's model. The ranges of the histograms from the AFM observation and current conductance were similar, with pore diameter being approximately $10 \mathrm{~nm}$ (e.g., $2-12 \mathrm{~nm}$ in Figure $5 \mathrm{~B}$ ). On the other hand, the pore size of $\mathrm{PFN}_{\mathrm{S}}(\sim 1.5 \mathrm{~nm}$ in diameter from the Hille equation) was too small to form the complete pore because the width of the PFN monomer was determined to be approximately $2.9 \mathrm{~nm}$ from the crystal structure. ${ }^{41}$

Recent studies reported that PFN cannot form a complete pore with a diameter of $<5 \mathrm{~nm}$ and instead forms incomplete (arc-shaped) pores. $^{48,49}$ Therefore, we modified the Hille equation for correct estimation of the size of incomplete pores. Specifically, we established a more precise model for the arcshaped pores, which is reported in detail in the Supporting Information. $\mathrm{PFN}_{\mathrm{S}}$ represented the constant current steps probably due to the monomer addition, as summarized as the histograms of conductance (Figure 5C) and its variation to the current steps (Figure 5D). Using our model based on the conductance, pore areas for $\mathrm{PFN}_{\mathrm{S}}$ were from $0.3-3 \mathrm{~nm}^{2}$ (smallest section) to $9-70 \mathrm{~nm}^{2}$ (largest section; see the
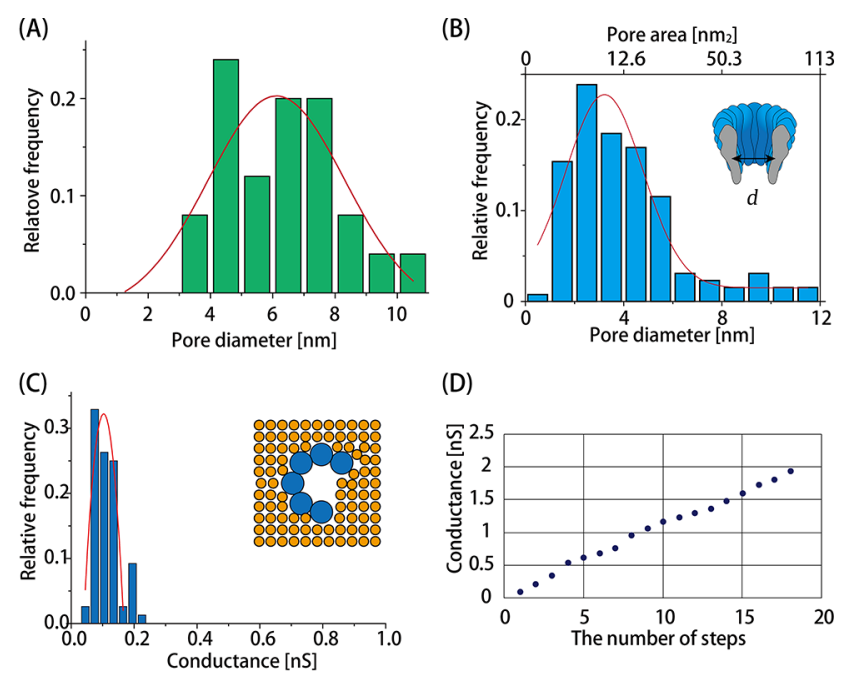

(D)
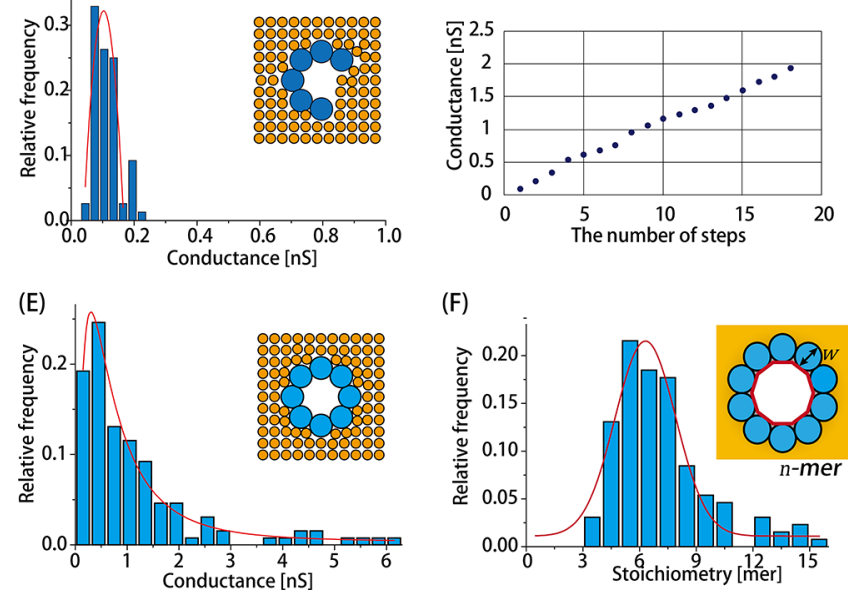

Figure 5. (A) A histogram of PFN pore diameter calculated from AFM imaging from a recent report. ${ }^{48}$ (B) A histogram of $\mathrm{PFN}_{\mathrm{L}}$ pore diameter and pore area calculated using the Hille equation from the results of channel current measurement. (C) A histogram of $\mathrm{PFN}_{S}$ pore conductance from channel current measurement. (D) Pore conductance of expanding $\mathrm{PFN}_{\mathrm{S}}$ pore. (E, F) A histogram of $\mathrm{PFN}_{\mathrm{L}}$ pore conductance from channel current measurement (E) and stoichiometry calculated from the pore diameter $(F)$.

Supporting Information). In our current recordings, two types of pore-forming behavior could be observed, suggesting that small pores may initially form and then become mature and complete pores.

Single-Protein Detection Using a PFN Nanopore. We attempted single-protein detection of GZB using a PFN nanopore. GZB is a serine protease with a diameter of approximately $5 \mathrm{~nm}$, and it has already been proposed that GZB would be transported through the PFN pores in a living system. $^{50}$ Figure $6 \mathrm{~A}-\mathrm{C}$ shows the typical current blocking events through PFN without or with GZB and the associated scatter plots. Interestingly, current blocking was only observed in $\mathrm{PFN}_{S}$ pores, whereas the pore size of $\mathrm{PFN}_{\mathrm{L}}$ was sufficient to allow the passage of GZB. A similar phenomenon was previously reported for a mitochondrial pore-forming protein. ${ }^{51,52} \mathrm{We}$ will discuss this phenomenon following the blocking event analysis on $\mathrm{PFN}_{\mathrm{S}}$.

The smallest value of the cross-sectional area of $\mathrm{PFN}_{\mathrm{s}}$ pores was lower than $3 \mathrm{~nm}^{2}$ for every step (see the Supporting Information). Therefore, we considered that the translocating events would reflect the translocation of unfolded GZB. A histogram of the blocking currents is presented in Figure 7A. Several groups of blocking events were observed and named peak 1 to peak 4 . These four different levels of current blocking imply four different translocation or pore-blocking states. The histograms of the duration (time of current blocking) of each peak are shown in Figures $7 \mathrm{~B}$, and the blocking current and duration are summarized in Table 4 . On the basis of these 

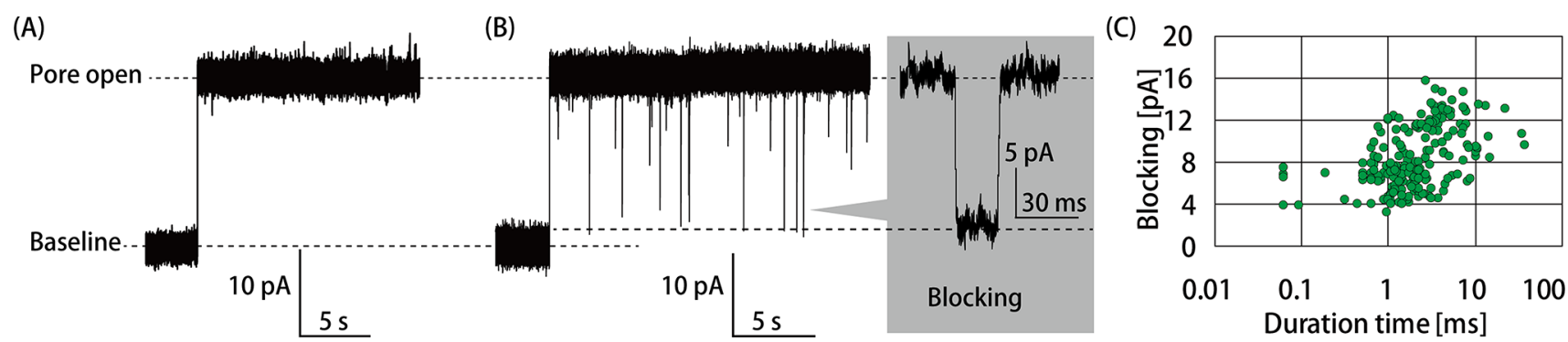

Figure 6. (A, B) The typical current-time traces of PFNs nanopore without (A) and with (B) GZB. (C) Scatter plots of current blocking and duration caused by GZB translocation. We defined the blocking current to be between the open and blocking levels.

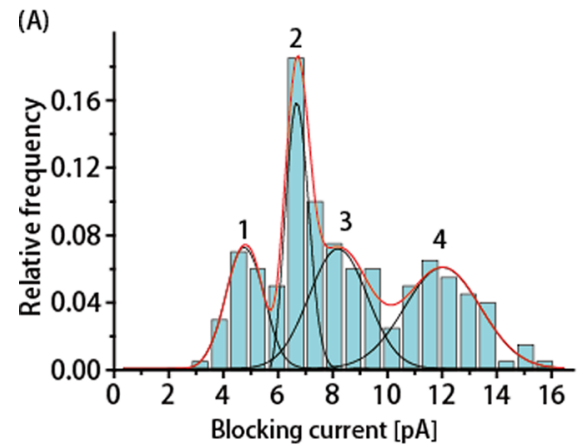

(C)

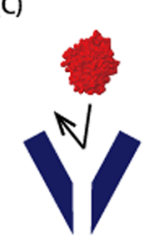

Peak 1

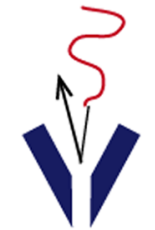

Peak 2

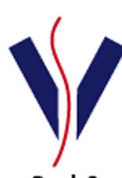

Peak 3
(B)
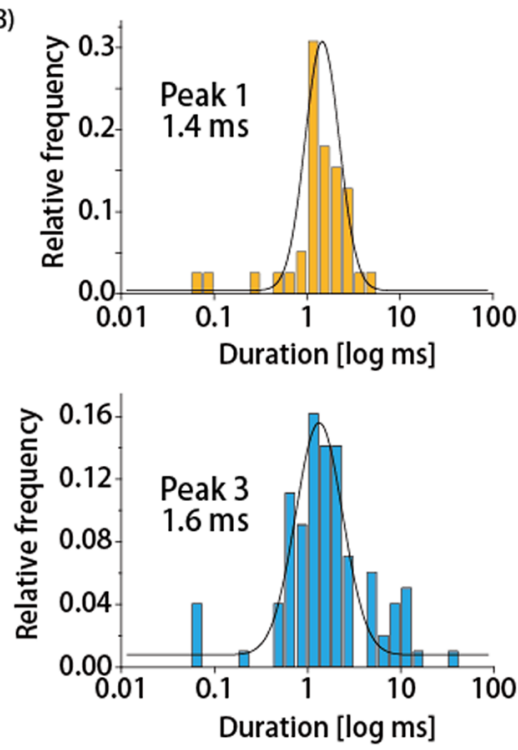
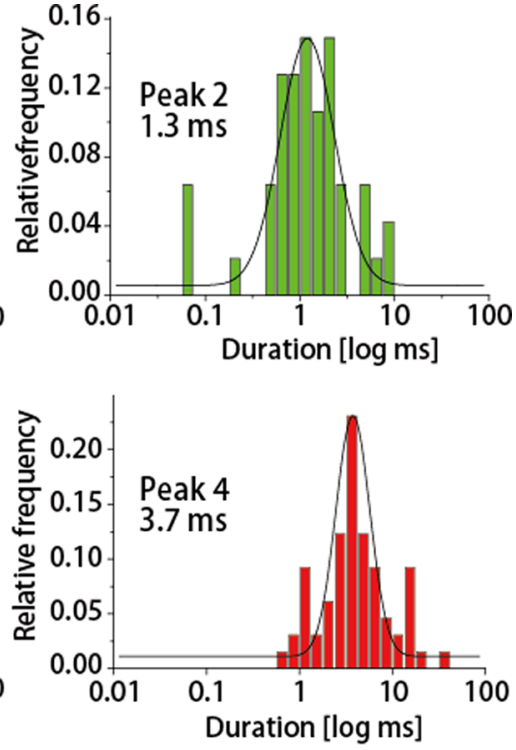

Figure 7. (A, B) Histograms of the blocking currents of GZB with $\mathrm{PFN}_{\mathrm{S}}(100 \mathrm{mV}$ application) (A) and duration of peaks 1-4 (B). (C) The possible current blocking model of each of the four peaks. Peaks 1-4 correspond to collision of folded protein, translocation of unfolded protein, translocation of unfolded protein, and folding and translocation of unfolded protein, respectively. These data were taken from three different PFN pores $(N=3)$.

Table 4. Blocking Currents (Mean) and Duration (Mean \pm SE) of Peaks $1-4^{a}$

\begin{tabular}{lllll} 
& \multicolumn{5}{c}{ peak no. } \\
\cline { 2 - 5 } & \multicolumn{1}{c}{1} & \multicolumn{4}{c}{2} & \multicolumn{1}{c}{3} & \multicolumn{1}{c}{4} \\
blocking [pA] & 4.8 & 6.7 & 8.2 & 12 \\
duration time [ms] & $1.4 \pm 0.3$ & $1.3 \pm 0.2$ & $1.6 \pm 0.9$ & $3.7 \pm 2.8$
\end{tabular}

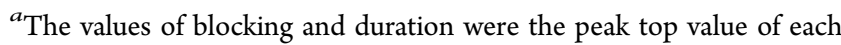
histogram, as shown in Figure 7A,B.

results, we propose a possible model for explaining peak 1 to peak 4 behavior as GZB binding or translocating to the PFN nanopore, as shown in Figure 7C. (1) Peak 1 showed the smallest current blocking and almost always had the shortest duration; this result may indicate the collision with the pore rumen of the folded GZB. (2) Peak 2 showed the second small blocking and almost always had the shortest duration; this behavior may reflect the collision with the pore rumen of the unfolded GZB. (3) Peak 3 represented the third current blocking state and had a slightly longer duration than peaks 1 and 2; it may represent translocation of the unfolded GZB through the pore. (4) Peak 4 showed the deepest current blocking and the longest duration, which was double that of peak 3; this can represent translocation with the unfolding process.
These are all possible models given that several reports have reported the translocation of unfolded proteins through biological nanopores. ${ }^{53}$ In addition, we examined the voltage dependence with changing from 100 to $120 \mathrm{mV}{ }^{54}$ Probably due to the faster events, the number of peaks declined in number to become three peaks (Figure S4). Because it was difficult to assign these three peaks against the previous four peaks, the mean duration was compared to the previous data. As the results, the mean duration became slightly shorter under $120 \mathrm{mV}(\sim 1.1 \mathrm{~ms})$.

We were not able to observe the translocation of GZB through the $\mathrm{PFN}_{\mathrm{L}}$ nanopore, as mentioned above. We considered that there were two possible explanations for this. One is that translocation of the folded GZB occurred too rapidly to be observed in our experimental conditions. A similar explanation was proposed in a previous paper. ${ }^{51}$ Another explanation is that the translocation of folded GZB requires cofactors, such as the translocon or supporting proteins. These cofactors may interact with GZB or PFN to support the transfer from one side to the other. This type of translocation has already been observed in parasite infections. ${ }^{55}$ In contrast to the $\mathrm{PFN}_{\mathrm{L}}$ nanopore, GZB in an unfolded state can pass through the $\mathrm{PFN}_{\mathrm{S}}$ nanopore upon the application of voltage. The blocking current signals can reflect the translocation behavior at the single-protein level. 


\section{CONCLUSION}

In summary, we explored large biological nanopores that were suitable for detecting a single protein from five different families of pore-forming proteins. Classification of the current signals of the protein nanopores was shown to be useful for estimating the adequacy of protein-targeted nanopore measurements. In this estimation, two types of pore formation were observed: additional or expanding pore formation. They could be discriminated using noise analysis. After this assessment, we selected the PFN nanopore because its size was the most suitable among the five nanopores. This nanopore showed two different types of pore formation: the additional and expanding models for large or small pore formation. Using PFN nanopores, we examined the availability of these nanopores for single-protein detection using GZB, which is $5 \mathrm{~nm}$ in diameter. The results showed that the blocking current signals were only observed in the $\mathrm{PFN}_{\mathrm{S}}$ pores, whereas the small pores were much smaller than the GZB protein. We concluded that unfolded GZB translocated through the $\mathrm{PFN}_{\mathrm{S}}$ pores, but some cofactors may be needed for transport through the $\mathrm{PFN}_{\mathrm{L}}$ pores. It should be noted that molecules that are smaller than nanopores will not always translocate through biological nanopores. For the extensive use of protein nanopores for nanopore sensing, further investigation of their selectivity will be required. Our analytical method of classification or noise analysis should be useful for such investigation.

\section{ASSOCIATED CONTENT}

\section{S Supporting Information}

The Supporting Information is available free of charge on the ACS Publications website at DOI: 10.1021/acs.analchem.7b01550.

Typical current-time traces for five nanopores; pore diameters calculated from Hille model; $I-V$ curve of a single $\mathrm{PFN}_{\mathrm{L}}$ pore; histogram of the blocking current signal of GZB with PFNs; model for low conductance perforin pores (PDF)

\section{AUTHOR INFORMATION}

\section{Corresponding Author}

*E-mail: rjkawano@cc.tuat.ac.jp.

\section{ORCID}

Ryuji Kawano: 0000-0001-6523-0649

\section{Author Contributions}

H.W. and R.K. conceived and designed the project. H.W. and N.T. performed the nanopore measurements. A.G. and M.C. conducted the theoretical analysis for the arc-shaped nanopores. $\mathrm{K}$. Tanaka and $\mathrm{K}$. Tsumoto expressed $\mathrm{FraC}$ protein and analyzed the structure. H.W, M.C., and R.K. analyzed the data and wrote the manuscript.

Notes

The authors declare no competing financial interest.

\section{ACKNOWLEDGMENTS}

This work was partly supported by KAKENHI (Grant Nos. 25708024, 15H00803, and 16H06043) from MEXT, Japan.

\section{REFERENCES}

(1) Gu, L. Q.; Braha, O.; Conlan, S.; Cheley, S.; Bayley, H. Nature 1999, 398 (6729), 686-690.
(2) Wanunu, M.; Dadosh, T.; Ray, V.; Jin, J. M.; McReynolds, L.; Drndic, M. Nat. Nanotechnol. 2010, 5 (11), 807-814.

(3) Kawano, R.; Osaki, T.; Sasaki, H.; Takinoue, M.; Yoshizawa, S.; Takeuchi, S. J. Am. Chem. Soc. 2011, 133 (22), 8474-8477.

(4) Hiratani, M.; Ohara, M.; Kawano, R. Anal. Chem. 2017, 89 (4), 2312-2317.

(5) Song, L. Z.; Hobaugh, M. R.; Shustak, C.; Cheley, S.; Bayley, H.; Gouaux, J. E. Science 1996, 274 (5294), 1859-1866.

(6) Deamer, D. W.; Branton, D. Acc. Chem. Res. 2002, 35 (10), $817-$ 825.

(7) Rosen, C. B.; Rodriguez-Larrea, D.; Bayley, H. Nat. Biotechnol. 2014, 32 (2), 179-181.

(8) Nivala, J.; Mulroney, L.; Li, G.; Schreiber, J.; Akeson, M. ACS Nano 2014, 8 (12), 12365-12375.

(9) Di Marino, D.; Bonome, E. L.; Tramontano, A.; Chinappi, M. J. Phys. Chem. Lett. 2015, 6 (15), 2963-2968.

(10) Asandei, A.; Schiopu, I.; Chinappi, M.; Seo, C. H.; Park, Y.; Luchian, T. ACS Appl. Mater. Interfaces 2016, 8 (20), 13166-13179.

(11) Kasianowicz, J. J.; Brandin, E.; Branton, D.; Deamer, D. W. Proc. Natl. Acad. Sci. U. S. A. 1996, 93 (24), 13770-13773.

(12) Branton, D.; Deamer, D. W.; Marziali, A.; Bayley, H.; Benner, S. A.; Butler, T.; Di Ventra, M.; Garaj, S.; Hibbs, A.; Huang, X. H.; Jovanovich, S. B.; Krstic, P. S.; Lindsay, S.; Ling, X. S. S.; Mastrangelo, C. H.; Meller, A.; Oliver, J. S.; Pershin, Y. V.; Ramsey, J. M.; Riehn, R.; Soni, G. V.; Tabard-Cossa, V.; Wanunu, M.; Wiggin, M.; Schloss, J. A. Nat. Biotechnol. 2008, 26 (10), 1146-1153.

(13) Han, A. P.; Schurmann, G.; Mondin, G.; Bitterli, R. A.; Hegelbach, N. G.; de Rooij, N. F.; Staufer, U. Appl. Phys. Lett. 2006, 88 (9), 093901.

(14) Garaj, S.; Hubbard, W.; Reina, A.; Kong, J.; Branton, D.; Golovchenko, J. A. Nature 2010, 467, 190-193.

(15) Wanunu, M. Phys. Life Rev. 2012, 9 (2), 125-158.

(16) Goyal, G.; Lee, Y. B.; Darvish, A.; Ahn, C. W.; Kim, M. J. Nanotechnology 2016, 27 (49), 495301.

(17) Balme, S.; Coulon, P. E.; Lepoitevin, M.; Charlot, B.; Yandrapalli, N.; Favard, C.; Muriaux, D.; Bechelany, M.; Janot, J. M. Langmuir 2016, 32 (35), 8916-8925.

(18) Funakoshi, K.; Suzuki, H.; Takeuchi, S. Anal. Chem. 2006, 78 (24), 8169-8174.

(19) Ying, Y. L.; Cao, C.; Long, Y. T. Analyst 2014, 139 (16), $3826-$ 3835.

(20) Faller, M.; Niederweis, M.; Schulz, G. E. Science 2004, 303 (5661), 1189-1192.

(21) Butler, T. Z.; Pavlenok, M.; Derrington, I. M.; Niederweis, M.; Gundlach, J. H. Proc. Natl. Acad. Sci. U. S. A. 2008, 105 (52), 2064720652.

(22) Iacovache, I.; De Carlo, S.; Cirauqui, N.; Dal Peraro, M.; van der Goot, F. G.; Zuber, B. Nat. Commun. 2016, 7, 12062.

(23) Pastoriza-Gallego, M.; Rabah, L.; Gibrat, G.; Thiebot, B. n. d.; van der Goot, F. o. G.; Auvray, L. c.; Betton, J.-M.; Pelta, J. J. Am. Chem. Soc. 2011, 133 (9), 2923-2931.

(24) Subbarao, G. V.; van den Berg, B. J. Mol. Biol. 2006, 360 (4), $750-759$.

(25) Alcaraz, A.; Ramirez, P.; Garcia-Gimenez, E.; Lopez, M. L.; Andrio, A.; Aguilella, V. M. J. Phys. Chem. B 2006, 110 (42), 2120521209.

(26) Chen, M.; Khalid, S.; Sansom, M. S. P.; Bayley, H. Proc. Natl. Acad. Sci. U. S. A. 2008, 105 (17), 6272-6277.

(27) Locher, K. P.; Rees, B.; Koebnik, R.; Mitschler, A.; Moulinier, L.; Rosenbusch, J. P.; Moras, D. Cell 1998, 95 (6), 771-778.

(28) Mohammad, M. M.; Iyer, R.; Howard, K. R.; McPike, M. P.; Borer, P. N.; Movileanu, L. J. Am. Chem. Soc. 2012, 134 (22), 95219531.

(29) Bayrhuber, M.; Meins, T.; Habeck, M.; Becker, S.; Giller, K.; Villinger, S.; Vonrhein, C.; Griesinger, C.; Zweckstetter, M.; Zeth, K. Proc. Natl. Acad. Sci. U. S. A. 2008, 105 (40), 15370-15375.

(30) Hoogerheide, D. P.; Gurnev, P. A.; Rostovtseva, T. K.; Bezrukov, S. M. Nanoscale 2017, 9 (1), 183-192. 
(31) Dgany, O.; Gonzalez, A.; Sofer, O.; Wang, W. X.; Zolotnitsky, G.; Wolf, A.; Shoham, Y.; Altman, A.; Wolf, S. G.; Shoseyov, O.; Almog, O. J. Biol. Chem. 2004, 279 (49), 51516-51523.

(32) Wang, H.-Y.; Li, Y.; Qin, L.-X.; Heyman, A.; Shoseyov, O.; Willner, I.; Long, Y.-T.; Tian, H. Chem. Commun. 2013, 49 (17), 1741-1743.

(33) Simpson, A. A.; Tao, Y. Z.; Leiman, P. G.; Badasso, M. O.; He, Y. N.; Jardine, P. J.; Olson, N. H.; Morais, M. C.; Grimes, S.; Anderson, D. L.; Baker, T. S.; Rossmann, M. G. Nature 2000, 408 (6813), 745-750.

(34) Geng, J.; Wang, S. Y.; Fang, H. M.; Guo, P. X. ACS Nano 2013, 7 (4), 3315-3323.

(35) Mueller, M.; Grauschopf, U.; Maier, T.; Glockshuber, R.; Ban, N. Nature 2009, 459 (7247), 726-730.

(36) Soskine, M.; Biesemans, A.; Moeyaert, B.; Cheley, S.; Bayley, H.; Maglia, G. Nano Lett. 2012, 12 (9), 4895-4900.

(37) Kawano, R.; Tsuji, Y.; Sato, K.; Osaki, T.; Kamiya, K.; Hirano, M.; Ide, T.; Miki, N.; Takeuchi, S. Sci. Rep. 2013, 3, 1995.

(38) Kawano, R.; Tsuji, Y.; Kamiya, K.; Kodama, T.; Osaki, T.; Miki, N.; Takeuchi, S. PLoS One 2014, 9 (7), e102427.

(39) Watanabe, H.; Kawano, R. Anal. Sci. 2016, 32 (1), 57-60.

(40) Hille, B. Ion channels of excitable membranes, 3rd ed.; Sinauer: Sunderland, Mass., 2001; p xviii, 814 p.

(41) Law, R. H. P.; Lukoyanova, N.; Voskoboinik, I.; Caradoc-Davies, T. T.; Baran, K.; Dunstone, M. A.; D’Angelo, M. E.; Orlova, E. V.; Coulibaly, F.; Verschoor, S.; Browne, K. A.; Ciccone, A.; Kuiper, M. J.; Bird, P. I.; Trapani, J. A.; Saibil, H. R.; Whisstock, J. C. Nature 2010, 468 (468), 447-451.

(42) Tanaka, K.; Caaveiro, J. M. M.; Morante, K.; Gonzalez-Manas, J. M.; Tsumoto, K. Nat. Commun. 2015, 6, 6337.

(43) Chui, J. K. W.; Fyles, T. M. Chem. Soc. Rev. 2012, 41 (1), 148175.

(44) Melo, M. N.; Ferre, R.; Castanho, M. Nat. Rev. Microbiol. 2009, 7 (3), 245-250.

(45) Cruciani, R. A.; Barker, J. L.; Durell, S. R.; Raghunathan, G.; Guy, H. R.; Zasloff, M.; Stanley, E. F. Eur. J. Pharmacol., Mol. Pharmacol. Sect. 1992, 226 (4), 287-296.

(46) Heins, E. A.; Siwy, Z. S.; Baker, L. A.; Martin, C. R. Nano Lett. 2005, 5 (9), 1824-1829.

(47) Sekiya, K.; Akagi, T.; Tatsuta, K.; Sakakura, E.; Hashikawa, T.; Abe, A.; Nagamune, H. Microbes Infect. 2007, 9 (11), 1341-1350.

(48) Leung, C.; Hodel, A. W.; Brennan, A. J.; Lukoyanova, N.; Tran, S.; House, C. M.; Kondos, S. C.; Whisstock, J. C.; Dunstone, M. A.; Trapani, J. A.; Voskoboinik, I.; Saibil, H. R.; Hoogenboom, B. W. Nat. Nanotechnol. 2017, 12 (5), 467-473.

(49) Praper, T.; Sonnen, A.; Viero, G.; Kladnik, A.; Froelich, C. J.; Anderluh, G.; Dalla Serra, M.; Gilbert, R. J. C. J. Biol. Chem. 2011, 286 (4), 2946-2955.

(50) Trapani, J. A.; Smyth, M. J. Nat. Rev. Immunol. 2002, 2 (10), 735-747.

(51) Shimizu, S.; Ide, T.; Yanagida, T.; Tsujimoto, Y. J. Biol. Chem. 2000, 275 (16), 12321-12325.

(52) Aksoyoglu, M. A.; Podgornik, R.; Bezrukov, S. M.; Gurnev, P. A.; Muthukumar, M.; Parsegian, V. A. Proc. Natl. Acad. Sci. U. S. A. 2016, 113 (32), 9003-9008.

(53) Cressiot, B.; Braselmann, E.; Oukhaled, A.; Elcock, A. H.; Pelta, J.; Clark, P. L. ACS Nano 2015, 9 (9), 9050-9061.

(54) Stefureac, R. I.; Trivedi, D.; Marziali, A.; Lee, J. S. J. Phys.: Condens. Matter 2010, 22 (45), 454133.

(55) Spielmann, T.; Gilberger, T. W. Trends Parasitol. 2015, 31 (10), 514-525. 